\title{
Short Combinatorial Proof that the DFJ Polytope is contained in the MTZ Polytope for the Asymmetric Traveling Salesman Problem
}

\author{
Mark Velednitsky * \\ UC Berkeley \\ marvel@berkeley.edu
}

\begin{abstract}
For the Asymmetric Traveling Salesman Problem (ATSP), it is known that the Dantzig-FulkersonJohnson (DFJ) polytope is contained in the Miller-Tucker-Zemlin (MTZ) polytope. The analytic proofs of this fact are quite long. Here, we present a proof which is combinatorial and significantly shorter by relating the formulation to distances in a modified graph.
\end{abstract}

Keywords - salesman, polytope, mtz, subtour, combinatorial

\section{Introduction}

The Asymmetric Traveling Salesman Problem (ATSP) on the graph $G=(V, A)$ is typically formulated as an Integer Program (IP) by assigning each arc $(i, j)$, of weight $c_{i j}$, a binary variable $x_{i j}$ indicating whether or not it participates in the tour:

$$
\begin{aligned}
& \operatorname{minimize} \sum_{(i, j) \in A} c_{i j} x_{i j} \\
& \begin{array}{cl}
\text { subject to } \sum_{j} x_{i j}=1 & \forall i \in V \\
\sum_{i} x_{i j}=1 & \forall j \in V
\end{array} \\
& \text { no sub-tours in }\left\{(i, j) \mid x_{i j}=1\right\} \\
& x_{i j} \in\{0,1\} \\
& \forall(i, j) \in A
\end{aligned}
$$

Several variants of the sub-tour elimination constraint (11) have been proposed. The DFJ constraints are:

$$
\sum_{i \in Q} \sum_{j \in Q} x_{i j} \leq|Q|-1
$$

for any $Q \subseteq\{2,3, \ldots, n\}$. The MTZ constraints introduce a new variable $u_{i}$ at each node $i \in V$ such that [5]:

$$
u_{i}-u_{j}+n x_{i j} \leq n-1 \forall(i, j) \in A
$$

The $u_{i}$ are meant to enumerate the order in which nodes appear in the tour. That is, $u_{i}=1$ for the first node, $u_{i}=2$ for the second, and so on.

\footnotetext{
*Fellow, National Physical Science Consortium
} 
The DFJ and MTZ polytopes are the feasible regions of the respective LP relaxations. It is known that the MTZ formulation produces a weaker LP relaxation. However, rigorous proofs of this fact are quite involved [2, 3, 4, 6].

Even though they are weaker, MTZ-like constraints have been applied to Vehicle Routing Problems and are popular for solving small instances of ATSP [1]. Having a concise proof of their weakness could be instructive for understanding the constraints, teaching them, and applying them elsewhere [7.

\section{Proof}

Theorem 1. The DFJ polytope is contained in the MTZ polytope.

Proof. Let $x_{i j}$ be feasible for formulation DFJ. We define a new graph $G$ where the arc weights are $(n-1)-$ $n x_{i j}$. We let $-u_{j}$ be the length of the shortest path from 1 to $j$ in $G$. We claim that these $u_{j}$ are well-defined and make the $u_{j}$ and $x_{i j}$ together satisfy formulation MTZ. To check that MTZ is satisfied, we write the shortest path condition in $G$ :

$$
-u_{j} \leq-u_{i}+(n-1)-n x_{i j} \Longrightarrow u_{i}-u_{j}+n x_{i j} \leq(n-1) .
$$

To confirm that the $u_{j}$ are well-defined, we need to prove there are no negative-cost cycles in $G$. Assume there is a negative cost cycle with edge set $C$ with node set $Q$ :

$$
\sum_{(i, j) \in C}\left((n-1)-n x_{i j}\right)<0 \Longrightarrow|Q|(n-1)-n \sum_{(i, j) \in C} x_{i j}<0 \Longrightarrow|Q| \frac{n-1}{n}<\sum_{(i, j) \in C} x_{i j} .
$$

But the conditions of formulation DFJ give us

$$
\sum_{(i, j) \in C} x_{i j} \leq|Q|-1
$$

This is a contradiction (since $|Q|=|C| \leq n$ ), so there are no negative cost cycles.

\section{References}

[1] Tolga Bektaş and Luis Gouveia. Requiem for the miller-tucker-zemlin subtour elimination constraints? European Journal of Operational Research, 236(3):820-832, 2014.

[2] Martin Desrochers and Gilbert Laporte. Improvements and extensions to the miller-tucker-zemlin subtour elimination constraints. Operations Research Letters, 10(1):27-36, 1991.

[3] Luis Gouveia and Jose Manuel Pires. The asymmetric travelling salesman problem and a reformulation of the miller-tucker-zemlin constraints. European Journal of Operational Research, 112(1):134-146, 1999.

[4] André Langevin, François Soumis, and Jacques Desrosiers. Classification of travelling salesman problem formulations. Operations Research Letters, 9(2):127-132, 1990.

[5] Clair E Miller, Albert W Tucker, and Richard A Zemlin. Integer programming formulation of traveling salesman problems. Journal of the ACM (JACM), 7(4):326-329, 1960.

[6] Manfred Padberg and Ting-Yi Sung. An analytical comparison of different formulations of the travelling salesman problem. Mathematical Programming, 52(1):315-357, 1991.

[7] Gabor Pataki. Teaching integer programming using the travleing salesman problem. SIAM Review, 45(1):116-123, 2003. 